\title{
Detection of Anti-Yellow Fever Virus Immunoglobulin M Antibodies at 3-4 Years Following Yellow Fever Vaccination
}

\author{
Katherine B. Gibney, $\uparrow$ Srilatha Edupuganti, $\uparrow$ Amanda J. Panella, Olga I. Kosoy, Mark J. Delorey, Robert S. Lanciotti, \\ Mark J. Mulligan, Marc Fischer, and J. Erin Staples* \\ Division of Vector-Borne Diseases, Centers for Disease Control and Prevention (CDC), Fort Collins, Colorado; Epidemic Intelligence \\ Service Program, CDC, Atlanta, Georgia; Department of Medicine, Division of Infectious Diseases, \\ Emory University School of Medicine, Atlanta, Georgia
}

\begin{abstract}
The duration of anti-yellow fever (YF) virus immunoglobulin M (IgM) antibodies following YF vaccination is unknown, making it difficult to interpret positive $\operatorname{IgM}$ antibody results in previously vaccinated travelers. We evaluated the frequency and predictors of YF IgM antibody positivity 3-4 years following YF vaccination. Twenty-nine (73\%) of 40 participants had YF IgM antibodies 3-4 years postvaccination. No demographic or exposure variables were predictive of YF IgM positivity. However, persons who were YF IgM positive at 3-4 years postvaccination had earlier onset viremia and higher neutralizing antibody geometric mean titers at 1 month and 3-4 years postvaccination compared with persons who were YF IgM negative. Detection of YF IgM antibodies several years postvaccination might reflect remote YF vaccination rather than recent YF vaccination or YF virus infection.
\end{abstract}

\section{INTRODUCTION}

Yellow fever (YF) virus, a mosquito-borne flavivirus, is endemic in tropical and subtropical areas of sub-Saharan Africa and South America. ${ }^{1,2}$ Humans can act as transient, amplifying hosts for YF virus and play an important role in its spread. In humans, the majority of YF virus infections are asymptomatic. Clinical disease ranges from a mild, undifferentiated febrile illness to severe jaundice and hemorrhage ${ }^{3}$; the case-fatality ratio of severe $\mathrm{YF}$ is $20-50 \%$. Because no treatment is available, prevention of infection through personal protective measures and vaccination is critical. Yellow fever vaccination is recommended for persons $\geq 9$ months of age who are traveling to or living in YF-endemic areas. ${ }^{1}$

The YF vaccine is a live-attenuated viral vaccine. Primary vaccinees typically develop a low level viremia, which abates as anti-YF virus immunoglobulin $\mathrm{M}$ (IgM) antibodies develop 4-7 days postvaccination. ${ }^{1}$ The $\operatorname{IgM}$ antibody levels usually peak by 2 weeks postvaccination and decline over several months. ${ }^{3,4}$ By 30 days postvaccination, $99-100 \%$ of vaccinees will have YF virus-specific neutralizing antibodies that can persist for decades. ${ }^{3,5,6}$

Testing of serum samples collected from ill travelers who have returned from YF-endemic areas periodically reveals YF IgM and neutralizing antibodies. Yellow fever IgM antibodies have been documented in serum up to 18 months postvaccination. ${ }^{4}$ In addition, several case investigations have identified YF IgM antibodies in the serum of ill travelers who had recently returned from YF-endemic areas and had received YF vaccine $>2$ years previously (CDC, unpublished data). In these cases, it was unclear whether the YF IgM antibodies resulted from recent infection with wild-type YF virus or another flavivirus, or prolonged production of YF IgM antibodies postvaccination. To aid in the interpretation of positive YF IgM serology in vaccinated persons, we evaluated the frequency and predictors of YF IgM positivity at 3-4 years following YF vaccination.

* Address correspondence to J. Erin Staples, Division of Vector-Borne Diseases, Centers for Disease Control and Prevention (CDC), 3156 Rampart Road, Fort Collins, CO 80521. E-mail: estaples@cdc.gov

$\dagger$ These authors contributed equally to this work.

\section{MATERIALS AND METHODS}

We enrolled 40 adults who received 17D-YF vaccine (Sanofi Pasteur, Swiftwater, PA) during a clinical trial conducted in 2006-2007 in Atlanta, GA (ClinicalTrials.gov identifier: NCT00254826). The purpose of the original clinical trial was to investigate the effect of coadministration of YF vaccine and immune globulin on the amount of YF vaccine virus present in the blood after vaccination and immune response to $\mathrm{YF}$ vaccine (Edupuganti $\mathrm{S}$, in press); YF $\operatorname{IgM}$ levels were not tested as part of this clinical trial. All subjects tested negative for West Nile, dengue, and YF virus $\mathrm{IgG}$ antibodies and YF virus-specific neutralizing antibodies before enrollment and were considered flavivirus-naive before YF vaccination. Viremia was detected in all but one of the participants. Testing for YF virus-specific neutralizing antibodies in samples collected in the first 3 months postvaccination occurred in batches with each patient's samples tested together (in parallel). Yellow fever virus-specific neutralizing antibodies developed in all participants by 14 days postvaccination and were present in all at 3 months postvaccination (last time point evaluated). No difference was noted in the viremia or immune response between participants who received YF vaccine alone and those who received YF vaccine with immune globulin (Edupuganti S, in press).

In 2010, at 3-4 years following YF vaccination, we collected serum and tested it for anti-YF virus antibodies by IgM antibody-capture enzyme-linked immunosorbent assay (MAC-ELISA) and YF virus-specific neutralizing antibodies by the plaque reduction neutralization test with a $90 \%$ cutoff value $\left(\mathrm{PRNT}_{90}\right)^{7,8}$ Testing of samples collected in 2010 occurred as a single batch for YF IgM and for YF virus-specific neutralizing antibodies. The YF IgM testing was only performed on samples collected at 3-4 years postvaccination. The PRNT testing of samples collected at 3-4 years postvaccination was not performed with earlier samples. We defined a positive $\mathrm{YF}$ IgM result as a positive to negative ratio $(\mathrm{P} / \mathrm{N})$ $\geq 3$ on MAC-ELISA and a negative YF IgM result as a $\mathrm{P} / \mathrm{N}$ ratio of $<3$. Seroprotection was defined as an $\mathrm{YF}$ virus $\mathrm{PRNT}_{90}$ titer $\geq 20$.

To determine potential predictors of YF IgM positivity at 3-4 years postvaccination, a standardized questionnaire was administered during the 2010 follow-up visit conducted in 
Atlanta, GA. Information was collected on demographics, underlying medical conditions, travel, and flaviviral disease or vaccination. Potential laboratory predictors of YF IgM positivity at 3-4 years postvaccination were evaluated including: 1) postvaccination viremia measured by a quantitative Taqman real-time reverse transcription-polymerase chain reaction [RT-PCR] ${ }^{9}$ on Days $0,1,2,3,5,7,9,11$, and 14 postvaccination; and 2) YF virus-specific neutralizing antibodies in serum collected on Days 2, 7, 9, 11, 14, 30, and 90 postvaccination. Categorical variables were compared using Fisher's exact tests and continuous variables using Wilcoxon rank sums or Student's $t$ tests. Persons with negative RT-PCR results were assigned a value of 1 for calculation of mean log viremia; negative PRNT results were assigned a value of 1 for calculation of geometric mean titer (GMT).

This follow-up study was approved by the Institutional Review Boards of Emory University and the Centers for Disease Control and Prevention (CDC) and participants provided informed consent before enrollment.

\section{RESULTS}

Of the 40 participants, 22 (55\%) were female (Table 1). The median age was 32 years (range 22-44 years) and the median time between YF vaccination and follow-up was 3.6 years (range 3.1-4.0 years). Study participants were generally healthy with only $2(5 \%)$ reporting an autoimmune disease (psoriasis and Raynaud's syndrome) and none reporting other potential immunosuppressive conditions or medications. No participant reported being diagnosed with a flaviviral infection since receiving YF vaccine. However, 25 (63\%) participants reported having traveled to a dengue-endemic country,

TABLE 1

Demographic and exposure history of adults receiving yellow fever (YF) vaccination, by $\mathrm{YF}$ immunoglobulin $\mathrm{M}(\mathrm{IgM})$ status at 34 years postvaccination*

\begin{tabular}{|c|c|c|c|c|}
\hline & \multicolumn{2}{|c|}{$\begin{array}{l}\text { YF IgM positive } \\
\quad(N=29)\end{array}$} & \multicolumn{2}{|c|}{$\begin{array}{l}\text { YF IgM negative } \\
\quad(N=11)\end{array}$} \\
\hline & No. & $(\%)$ & No. & (\%) \\
\hline Female & 14 & (48) & 8 & (73) \\
\hline \multicolumn{5}{|l|}{ Age group at follow-up (years) } \\
\hline $20-29$ & 10 & (34) & 6 & $(55)$ \\
\hline $30-39$ & 15 & $(52)$ & 3 & (27) \\
\hline $40-49$ & 4 & (14) & 2 & (18) \\
\hline$\geq 50$ & 0 & $(0)$ & 0 & $(0)$ \\
\hline \multicolumn{5}{|c|}{ Self-reported medical conditions at follow-up } \\
\hline Autoimmune disease & 1 & (3) & 1 & (9) \\
\hline Hypertension & 2 & (7) & 0 & (0) \\
\hline Dyslipidemia & 1 & (3) & 0 & (0) \\
\hline Hypothyroidism & 0 & (0) & 1 & (9) \\
\hline \multicolumn{5}{|l|}{ Lot of YF vaccine received } \\
\hline Lot 1 & 27 & (93) & 10 & (91) \\
\hline Lot 2 & 2 & (7) & 1 & (9) \\
\hline $\begin{array}{l}\text { Immune globulin coadministered } \\
\text { with vaccine }\end{array}$ & 15 & $(52)$ & 6 & $(55)$ \\
\hline \multicolumn{5}{|l|}{ Postvaccination exposures } \\
\hline Travel to YF endemic country & 5 & (17) & 4 & (36) \\
\hline Travel to dengue endemic country & 16 & (55) & 9 & $(82)$ \\
\hline Japanese encephalitis vaccine & 2 & (7) & 0 & (0) \\
\hline Tick-borne encephalitis vaccine & 0 & (0) & 0 & (0) \\
\hline
\end{tabular}

$9(23 \%)$ traveled to an YF-endemic country, and $2(5 \%)$ received Japanese encephalitis vaccine.

At 3-4 years postvaccination, 29 (73\%) participants were YF IgM positive with a median $\mathrm{P} / \mathrm{N}$ ratio of 5.1 (range 3.013.4) and 11 (27\%) were YF IgM negative with a median $\mathrm{P} / \mathrm{N}$ of 1.6 (range 0.9-2.5). No demographic or exposure variables, including vaccine lot used or immune globulin coadministration, were predictive of being YF IgM positive (Table 1). Persons who were YF IgM positive at 3-4 years postvaccination had an earlier detectable viremia (median 3 days postvaccination, range 1-7) than those who were YF IgM negative (median 5 days postvaccination, range $3-7)(P<0.05)$. The YF IgM-positive participants had a median peak viremia of 2,253 genomes/mL (range 21-51,931) compared with 298 genomes/mL (range undetectable-41,244) in YF IgMnegative participants. This difference was not statistically significant (ratio of genomes $/ \mathrm{mL}=7.36 ; 95 \%$ confidence intervals $[95 \% \mathrm{CI}]=0.9-54.5)$. No difference was detected in the time to develop seroprotective YF virus-specific neutralizing antibody levels between the YF IgM-positive group (median 11 days postvaccination, range 9-14) and the YF IgM negative group (median 11 days postvaccination, range 9-15) $(P=$ 0.33 ). However, at 1 month postvaccination, YF virus-specific neutralizing antibodies were significantly higher in the YF IgM positive group (GMT 3,843, range 320-20,480) versus the YF IgM negative group (GMT 1,060, range 40-10,240) (ratio of $\mathrm{GMTs}=10.3$; 95\% CI $=1.1-11.9$ ) (Figure 1). At 3-4 years postvaccination, YF virus-specific neutralizing antibodies were still significantly higher in the YF IgM positive group (GMT 86, range 40-320) versus the YF IgM negative group (GMT 27, range undetectable-320) (ratio of GMTs $=10.0 ; 95 \% \mathrm{CI}=1.1-$ 11.6). Three ( $8 \%)$ persons lacked seroprotective neutralizing antibodies at 3-4 years postvaccination; all were YF IgM negative. Finally, there was no correlation found between YF IgM $\mathrm{P} / \mathrm{N}$ ratios at 3-4 years postvaccination and time to detectable viremia, peak viremia level, neutralizing titers at 30 days, or neutralizing titers at 3-4 years postvaccination.

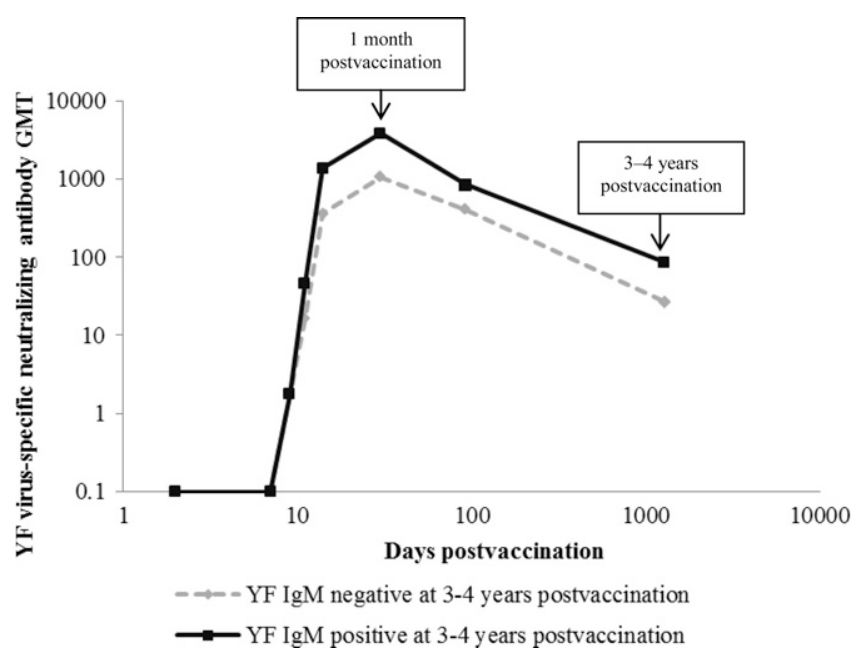

FIGURE 1. Yellow fever (YF) virus-specific neutralizing antibody geometric mean titers (GMT) following YF vaccination, by YF virus immunoglobulin M (IgM) status at 3-4 years postvaccination. Serum collected at 2, 7, 9, 11, and 14 days, 1 month, 3 months, and 34 years postvaccination 


\section{DISCUSSION}

Detection of anti-YF virus IgM antibodies is traditionally used to diagnose recent YF virus infection. The finding of YF $\mathrm{IgM}$ antibodies in almost three-quarters of primary YF vaccinees at 3-4 years postvaccination affects this interpretation and could potentially impact both the public health response and clinical management of a patient. Identification of YF IgM antibodies in a symptomatic person in or near an YFendemic area often triggers an $\mathrm{YF}$ vaccination campaign and vector control efforts. In non-endemic areas with vectors capable of transmitting YF virus, such as the United States, ${ }^{10}$ detection of YF IgM antibodies in an ill traveler could indicate potential importation of YF virus and the need to monitor for autochthonous transmission. If YF $\operatorname{IgM}$ antibodies represent vaccination rather than disease, an alternative etiology for the traveler's illness should then be sought and clinical management may need to be altered. In these situations, obtaining an YF vaccine history becomes essential for appropriately interpreting and acting on positive YF IgM results.

At least three previous studies have documented YF IgM antibodies from 2 to 18 months postvaccination, although none have documented the cause of prolonged IgM production. 411,12 Two of these studies only evaluated a total of three persons $>2$ months postvaccination and all were $\operatorname{IgM}$ positive. ${ }^{4,12}$ In the largest of the three studies, $13(43 \%)$ of 30 primary YF vaccinees in Brazil had detectable YF IgM antibodies at 100-349 days postvaccination. ${ }^{11}$ The lower proportion of vaccinees with YF IgM positivity in Brazil compared with our study might be caused by prior flaviviral infection in the Brazilian subjects, as vaccinees with preexisting dengue $\mathrm{IgG}$ antibodies were less likely to develop YF IgM antibodies following vaccination. This is consistent with previous studies that have found IgM antibodies may not develop after secondary flavivirus infections, or may develop more slowly or persist for a shorter period of time when compared with primary infections. ${ }^{13-15}$

There are several possible explanations for the presence of YF IgM antibodies in the serum of persons 3-4 years postvaccination. First, persistent infection with YF vaccine virus may have resulted in prolonged $\operatorname{IgM}$ antibody synthesis. ${ }^{15}$ In one recent study of 44 travelers, YF vaccine virus RNA was detected in the urine of two subjects at 21 days and 6 months postvaccination. ${ }^{16}$ However, YF vaccine virus RNA was not detected in urine of the remaining 42 persons tested from 0-11 months postvaccination. Persistent infection has also been shown in animal studies, including detection of intracerebral YF vaccine virus in three of eight monkeys at 63-159 days post-intracerebral inoculation and detection of YF virus-specific antigen in brains of mice at 7 weeks postintracerebral inoculation of YF vaccine virus. ${ }^{17,18}$ Second, continuous presentation of viral particles by antigen presenting cells could lead to antibody persistence as was documented in a study that used synthetic nanoparticles to mimic YF vaccine in the activation of dendritic cells by multiple toll-like receptors. ${ }^{19}$ Viral or antigen persistence also has been suggested as a possible mechanism for the prolonged production of IgM antibodies seen with other viruses, such as chikungunya, Japanese encephalitis, and rubella. ${ }^{20-22}$ A third possible explanation for prolonged $\operatorname{IgM}$ antibody synthesis is an incomplete class switching either caused by alterations in the vaccine virus because of the attenuation process or poten- tial interference of the vaccine virus in the regulation or recognition of molecules needed for class switching. ${ }^{23,24}$ Finally, subsequent exposure to YF virus or a related flavivirus could have caused IgM antibodies to persist or reform after infection. ${ }^{25}$ We did not, however, find a significant association between being YF IgM positive and potential subsequent flavivirus exposure (e.g., travel to a YF or dengue-endemic area or flavivirus vaccination).

Although the reason for YF IgM being found in a large proportion of YF vaccine recipients several years after vaccination is unknown, our data suggest there is correlation between YF IgM persistence and the initial viremia and immune response. We found that individuals who were YF IgM positive at 3-4 years postvaccination had earlier onset viremia and higher neutralizing antibody titers at 1 month and 3-4 years postvaccination compared with those who were YF IgM negative. Peak viremia, however, was not statistically significant between the two groups. The lack of a correlation between peak viremia and antibody response was also observed in a previous study. ${ }^{26}$

Our study has certain limitations. Study participants were healthy young adults who lived in the United States. Thus, they might not be representative of all travelers or persons living in YF-endemic areas, particularly in terms of exposure to other flaviviruses either before or after vaccination. Although no differences were detected in the exposure histories between persons who were YF IgM positive and negative, we had limited travel information and therefore were unable to quantify their potential risk of exposure to YF virus or a related flavivirus. Additionally, we did not collect specific information about where the participants resided between vaccination and their follow-up visit. Therefore, it was not possible to determine if participants had different potential exposure risk for domestic flaviviruses. Finally, this cohort was not tested for YF IgM antibodies between vaccination and 3-4 years postvaccination. Therefore, we cannot determine if detection of YF IgM represents persistence versus fluctuations in YF IgM antibody synthesis postvaccination.

Our results suggest detection of YF IgM in vaccinated persons might represent previous YF vaccination rather than recent infection with wild-type YF virus. To evaluate for a recent YF infection versus previous vaccination, clinicians should obtain a vaccine history in persons being evaluated for possible YF disease. Persons with a clinically compatible illness can be evaluated for infection by obtaining an acute serum and testing for YF virus RNA by RT-PCR. Alternatively, paired specimens can be obtained to look for a 4-fold rise in antibody titers between acute and convalescent samples. Taking into account the vaccine history of someone suspected to have YF disease is necessary to accurately interpret YF serologic results, manage the case clinically, and develop an effective public health response.

Received March 21, 2012. Accepted for publication August 27, 2012. Published online October 29, 2012

Acknowledgments: We thank Beverly Weaver and Nayoka Rose for assistance with data collection; Benton Lawson, Seema Garg, and Angela Caliendo for original RT-PCR work and data; Peggy Collins for assistance with database management; and Christine Klein for assistance with data entry.

Financial support: Personnel at the Hope Clinic were partially funded through the Georgia Research Alliance. 
Disclaimer: The findings and conclusions of this report are those of the authors and do not necessarily represent the views of the Centers for Disease Control and Prevention.

Authors' addresses: Katherine Gibney, Department of Epidemiology and Preventive Medicine, Monash University, Victoria, Australia, E-mail: Katherine.Gibney@monash.edu. Srilatha Edupuganti and Mark J. Mulligan, Decatur, GA, E-mails: edupug@emory.edu and mark.mulligan@emory.edu. Amanda J. Panella, Olga I. Kosoy, Mark J. Delorey, Robert S. Lanciotti, Marc Fischer, and J. Erin Staples, Fort Collins, CO, E-mails: apanella@cdc.gov, okosoy@cdc.gov, mdelorey@ cdc.gov, rslanciotti@cdc.gov,mfischer@cdc.gov, and estaples@cdc.gov.

\section{REFERENCES}

1. Centers for Disease Control and Prevention, 2010. Yellow fever vaccine: recommendations of the Advisory Committee on Immunization Practices (ACIP). MMWR Recomm Rep 59: 1-27.

2. Jentes ES, Poumerol G, Gershman MD, Hill DR, Lemarchand J, Lewis RF, Staples JE, Tomori O, Wilder-Smith A, Monath TP, 2011. The revised global yellow fever risk map and recommendations for vaccination, 2010: consensus of the informal WHO working group on geographic risk of yellow fever. Lancet Infect Dis 11: 622-632.

3. Monath TP, Teuwen D, Cetron M, 2008. Yellow fever vaccine. Plotkin S, Orenstein W, Offit P, eds. Vaccines. Fifth edition. China: Elsevier, 959-1055.

4. Monath TP, 1971. Neutralizing antibody responses in the major immunoglobulin classes to yellow fever 17D vaccination of humans. Am J Epidemiol 93: 122-129.

5. Groot H, Riberiro RB, 1962. Neutralizing and hemagglutinationinhibiting antibodies to yellow fever 17 years after vaccination with 17D vaccine. Bull World Health Organ 27: 699-707.

6. Poland JD, Calisher CH, Monath TP, Downs WG, Murphy K, 1981. Persistence of neutralizing antibody 30-35 years after immunization with 17D yellow fever vaccine. Bull World Health Organ 59: 895-900.

7. Martin DA, Muth DA, Brown T, Johnson AJ, Karabatsos N, Roehrig JT, 2000. Standardization of immunoglobulin M capture enzyme-linked immunosorbent assays for routine diagnosis of arboviral infections. J Clin Microbiol 38: 1823-1826.

8. Beaty BJ, Calisher CH, Shope RE, 1995. Arboviruses. Lennette EH, Lennette DA, Lennette ET, eds. Diagnostic Procedures for Viral, Rickettsial and Chlamydial Infections. Seventh edition. Washington, DC: American Public Health Association, 189-212.

9. Miller JD, van der Most RG, Akondy RS, Glidewell JT, Albott S, Masopust D, Murali-Krishna K, Mahar PL, Edupuganti S, Lalor S, Germon S, Del Rio C, Mulligan MJ, Staprans SI, Altman JD, Feinberg MB, Ahmed R, 2008. Human effector and memory CD8+ T cell responses to smallpox and yellow fever vaccines. Immunity 28: 710-722.

10. Darsie R, Ward R, 2005. Identification and Geographical Distribution of the Mosquitoes of North America, North of Mexico. Gainesville, FL: University Press of Florida.

11. de Melo AB, da Silva Mda P, Magalhães MC, Gonzales Gil LH, Freese de Carvalho EM, Braga-Neto UM, Bertani GR, Marques ET Jr, Cordeiro MT, 2011. Description of a prospective 17DD yellow fever vaccine cohort in Recife, Brazil. Am J Trop Med Hyg 85: 739-747.
12. Nogueira R, Schatzmayr H, Miagostovich M, Cavalcanti S, de Carvalho R, 1992. Use of MAC-ELISA for evaluation of yellow fever vaccination. Rev Inst Med Trop Sao Paulo 34: 447-450.

13. Innis BL, Nisalak A, Nimmannitya S, Kusalerdchariya S, Chongswasdi V, Suntayakorn S, Puttisri P, Hoke CH, 1989. An enzyme-linked immunosorbent assay to characterize dengue infections where dengue and Japanese encephalitis co-circulate. Am J Trop Med Hyg 40: 418-427.

14. Lhuillier M, Sarthou J-L, 1983. Anti-yellow fever IgM in the diagnosis and epidemiological surveillance of yellow fever. Ann Virol 134E: 349-359.

15. Kuno G, 2001. Persistence of arboviruses and antiviral antibodies in vertebrate hosts: its occurrence and impacts. Rev Med Virol 11: $165-190$

16. Martínez MJ, Vilella A, Pumarola T, Roldan M, Sequera VG, Vera I, Hayes EB, 2011. Persistence of yellow fever vaccine RNA in urine. Vaccine 29: 3374-3376.

17. Penna HA, Bittencourt A, 1943. Persistence of yellow fever virus in brains of monkeys immunized by cerebral inoculation. Science 97: 448-449.

18. Gould EA, Buckley A, Cane PA, Higgs S, Cammack N, 1989. Use of a monoclonal antibody specific for wild-type yellow fever virus to identify a wild-type antigenic variant in 17D vaccine pools. J Gen Virol 70: 1889-1894.

19. Kasturi SP, Skountzou I, Albrecht RA, Koutsonanos D, Hua T, Nakaya HI, Ravindran R, Stewart S, Alam M, Kwissa M, Villinger F, Murthy N, Steel J, Jacob J, Hogan RJ, GarcíaSastre A, Compans R, Pulendran B, 2011. Programming the magnitude and persistence of antibody responses with innate immunity. Nature 470: 543-547.

20. Meurman $\mathrm{OH}, 1978$. Persistence of immunoglobulin $\mathrm{G}$ and immunoglobulin $\mathrm{M}$ antibodies after postnatal rubella infection determined by solid-phase radioimmunoassay. J Clin Microbiol 7: 34-38.

21. Edelman R, Schneider RJ, Vejjajiva A, Pornpibul R, Voodhikul $\mathrm{P}, 1976$. Persistence of virus-specific IgM and clinical recovery after Japanese encephalitis. Am J Trop Med Hyg 25: 733-738.

22. Malvy D, Ezzedine K, Mamani-Matsuda M, Autran B, Toluo H, Receveur MC, Pistone T, Rambert J, Moynet D, Mossalayi D, 2009. Destructive arthritis in a patient with chikungunya virus infection with persistent specific IgM antibodies. BMC Infect Dis 9: 200-206.

23. McAdam AJ, Farkash EA, Gewurz BE, Sharpe AH, 2000. B7 costimulation is critical for antibody class switching and CD8+ cytotoxic T-lymphocyte generation in the host response to vesicular stomatitis virus. J Virol 74: 203-208.

24. Fuse S, Obar JJ, Bellfy S, Leung E, Zhang W, Usherwood EJ, 2006. CD80 and CD86 control antiviral CD8+ T-cell function and immune surveillance of murine gammaherpesvirus 68 . J Virol 80: 9159-9170.

25. Makino Y, Tadano M, Saito M, Maneekarn N, Sittisombut N, Sirisanthana V, Poneprasert B, Fukunaga T, 1994. Studies on serological cross-reaction in sequential flavivirus infections. Microbiol Immunol 38: 951-955.

26. Reinhardt B, Jaspert R, Niedrig M, Kostner C, L'age-Stehr J, 1998. Development of viremia and humoral and cellular parameters of immune activation after vaccination with yellow fever virus strain 17D: a model of human flavivirus infection. J Med Virol 56: 159-167. 